\title{
The (CA) polymorphism of the TNFR2 gene is associated with peak bone density in Chinese nuclear families
}

Received: 27 December 2004/ Accepted: 18 March 2005/Published online: 11 May 2005

(C) The Japan Society of Human Genetics and Springer-Verlag 2005

\begin{abstract}
Low peak bone density (PBD) in adulthood is an important determinant of osteoporotic fracture (OF) in the elderly. The tumor necrosis factor receptor 2 (TNFR2) gene has been considered as an important candidate gene for PBD due to its important role in bone turnover. In this study, we recruited a total of 1,263 subjects from 402 Chinese nuclear families composed of both parents and at least one daughter, and tested the association of the $(\mathrm{CA})_{\mathrm{n}}$ polymorphism in intron 4 of the TNFR2 gene with PBD using a more contemporary quantitative transmission disequilibrium test (QTDT). Significant within-family association was detected between the CA16 allele and bone mineral density (BMD) at the lumbar spine with the $P$-value of 0.005 after permutations, which is still significant after correction for multiple testing. Some evidence of totalfamily association between the CA16 allele and lumbar spine BMD was found $(P=0.021)$, although the significant level did not reach the empirical threshold
\end{abstract}

H. Xu · S.-F. Lei $\cdot$ M.-X. Li $\cdot$ X. Sun $\cdot$ F.-Y. Deng $\cdot$

D.-K. Jiang $\cdot$ H.-W. Deng $(\triangle)$

Laboratory of Molecular and Statistical Genetics,

College of Life Sciences, Hunan Normal University,

Changsha, Hunan, 410081, People's Republic of China

E-mail: hwdeng@hunnu.edu.cn

Tel.: + 86-731-8872791

Fax: + 86-731-8872791

H. Xu

Department of Physiology, Jiangxi Medical College,

Nanchang, Jiangxi, 330006, People's Republic of China

L.-J. Zhao · H.-W. Deng

Osteoporosis Research Center and

Department of Biomedical Sciences,

Creighton University Medical Center,

601 N. 30th St., Suite 6787,

Omaha, NE 68131, USA

H.-W. Deng

The Key Laboratory of Biomedical Information

Engineering of Ministry of Education and

Institute of Molecular Genetics,

Xi'an Jiaotong University, Xi'an,

710049, People's Republic of China
$(P \leq 0.007)$. About $3.14 \%$ of lumbar spine BMD variation can be explained by the CA16 allele. In summary, our results suggest that the TNFR2 gene may play an important role in determining lumbar spine BMD variation in Chinese women.

Keywords Association. Tumor necrosis factor receptor 2 gene $\cdot$ Peak bone density $\cdot$ Transmission disequilibrium test

\section{Introduction}

Osteoporotic fracture (OF) is a serious health problem in our aging society. Low peak bone density (PBD) in adulthood is an important determinant factor of OF in the elderly. PBD has been demonstrated to be under strong genetic control (Deng et al. 1999). Several lines of evidence suggested that the tumor necrosis factor receptor 2 (TNFR2) gene as a strong candidate gene for PBD. TNFR2 can regulate the effects of TNF on osteoclastogenesis (Abu-Amer et al. 2000). TNFR2 gene is located in $1 \mathrm{p} 36.2$, where the potential quantitative trait loci for the variation of bone mineral density (BMD) at the hip were identified (Devoto et al. 2001). Studies in some populations have shown an association between the TNFR2 gene and BMD variation (Spotila et al. 2000, 2003; Albagha et al. 2002; Tasker et al. 2004). However, little is known about the effect of the TNFR2 gene on BMD variation in Chinese. In this study, we tested the association of the $(\mathrm{CA})_{\mathrm{n}}$ polymorphism in intron 4 of the TNFR2 gene with PBD in 402 Chinese nuclear families using quantitative transmission disequilibrium test (QTDT) (Abecasis et al. 2000).

\section{Subjects and methods}

A total of 402 nuclear families containing 1,263 subjects were recruited from a local population of Shanghai, P.R. 
China. They were all of the Chinese Han ethnicity, which is greater than $93 \%$ of the total Chinese population. Each nuclear family includes both parents and at least one healthy pre-menopausal daughter aged 19.344.0 years, when the Chinese female may achieve and maintain their PBD (19-44 years) (Yao et al. 2001). There were $86.8,12.4,0.5$ and $0.3 \%$ of nuclear families with one, two, three and four daughters, respectively. For each subject, BMDs $\left(\mathrm{g} / \mathrm{cm}^{2}\right)$ at the lumbar spine 1-4 (LS) and femoral neck (FN) were measured by a Hologic QDR2000+ dual-energy X-ray absorptiometry (DEXA) scanner (Hologic Inc., Bedford, MA, USA). The coefficient of variation (CV) values for repeated BMD measurements at the LS and FN were 0.9 and $1.93 \%$, respectively. Informed consent was obtained from each subject. Exclusion criteria were detailed earlier by Deng et al. (2002) to minimize any known potential confounding factors on bone and calcium metabolism.

Genomic DNA was isolated and the $(\mathrm{CA})_{\mathrm{n}}$ polymorphism of the TNFR2 gene was amplified using the polymerase chain reaction (PCR) with previously described primers (Spotila et al. 2000). PCR products were separated by electrophoresis on an ABI 377DNA Analyzer (Applied Biosytems, Foster City, CA, USA). Data were analyzed using software of GENESCAN and GENOTYPER (Applied Biosystems) and GenoDB ( $\mathrm{Li}$ et al. 2001). Alleles were termed as the number of CA repeats. The program PedCheck was employed for verifying Mendelian inheritance of all the marker alleles within the nuclear families (O'Connell and Weeks 1998).

Statistical Analysis System (SAS Institute, Cary, NC, USA) was used to analyze the basic statistics and to test the normal distribution of the bone phenotype data. Tests of population stratification, total-family association and within-family association of the $(\mathrm{CA})_{\mathrm{n}}$ polymorphism with BMD at the LS and FN were performed in the QTDT program (http://www.sph.umich.edu/csg/ abecasis/QTDT/). QTDT program was derived from the transmission disequilibrium test (TDT) program. TDT program was originally developed for qualitative traits, using a conventional $\chi^{2}$ test to compare the frequencies of the alleles transmitted from heterozygous parents to affected children relative to the frequencies of the untransmitted alleles (Spielman et al. 1993). QTDT extended methods to analyze quantitative traits, such as BMD (Abecasis et al. 2000). Both the original TDT and QTDT are robust to population admixture/stratification (Spielman et al. 1993; Abecasis et al. 2000).

In the QTDT analysis, we performed allele-wise tests for the four common alleles with the frequency above $5 \%$ based on the "common allele-common disease" hypothesis (Lander 1996). To avoid the multiple-testing problem, 1,000 permutation tests were performed to establish an empirical threshold (McIntyre et al. 2000), which was $P \leq 0.007$ for individual test to achieve a global significance level of 0.05 for each test in the present study.

\section{Results and discussion}

The basic characteristics of the study subjects are summarized in Table 1 . Ten alleles of the $(\mathrm{CA})_{\mathrm{n}}$ polymorphism were observed, with the number of CA repeats ranging from 10 to 19 . Allele frequencies of the $(\mathrm{CA})_{n}$ polymorphism in our sample are shown in Fig. 1. Four major alleles with frequency $>5 \%$, CA11, CA15, CA16 and CA17, accounted for $92.2 \%$.

Results of the QTDT analyses are summarized in Table 2. Both within-family and total-family associations are reliable since no population stratification was detected in our samples $(P>0.10)$. At the nominal significance level of 0.05 , the CA16 allele was shown within-family and total-family associations with LS BMD, with the $P$-values of 0.009 and 0.021 , respectively. When the multiple testing corrections were taken into account, the permutation result of within-family association between the CA16 allele and LS BMD remains significant $(P=0.005)$. The CA16 allele accounted for $3.14 \%$ of LS BMD variation, with carriers of the CA16 allele having, on average, $2.57 \%$ lower LS BMD than those without this allele. Moreover, since the age span of the offspring in our sample is somewhat broad (19.344.0 ), analyses excluding 30 subjects in young or older extremities (i.e., three subjects under age 20 years, and 27 subjects older than 40 years) were also performed. After excluding these 30 extremities, the results were more prominent and were consistent with those analyzed by the whole group (Table 2).

Our study first studied and found that the TNFR2 gene might be associated with LS BMD in Chinese women. This association, however, was not shown in the studies of Spotila et al. (2000, 2003). The different findings may be attributed to inter-study differences. In addition to the different analytical approaches applied in different studies, the different study subjects, i.e., healthy Chinese Han ethnic pre-menopausal women in our study and subjects including both male and female drawn from different ethnic backgrounds or only from European descent in Spotila et al. studies (2000, 2003), may also contribute to the inconsistent results.

The potential limitation is that due to budget limit, we selected only one polymorphism of the TNFR2 gene

Table 1 Characteristics of the study subjects. The BMD values (mean $\pm \mathrm{SD}$ ) are unadjusted raw values at the lumbar spine and femoral neck. Phenotypes are not available for 15 fathers and 14 mothers

\begin{tabular}{lccc}
\hline & $\begin{array}{l}\text { Father group } \\
(n=387)\end{array}$ & $\begin{array}{l}\text { Mother group } \\
(n=388)\end{array}$ & $\begin{array}{l}\text { Offspring group } \\
(n=459)\end{array}$ \\
\hline Age (year) & $62.4 \pm 6.6$ & $59.2 \pm 6.5$ & $31.4 \pm 5.8$ \\
Height $(\mathrm{cm})$ & $166.2 \pm 6.1$ & $154.6 \pm 5.6$ & $159.9 \pm 5.2$ \\
Weight $(\mathrm{kg})$ & $68.5 \pm 10.0$ & $59.3 \pm 8.6$ & $55.1 \pm 8.0$ \\
BMD $\left(\mathrm{g} / \mathrm{cm}^{2}\right)$ & & & \\
Lumbar spine & $0.931 \pm 0.149$ & $0.813 \pm 0.152$ & $0.960 \pm 0.102$ \\
Femoral neck & $0.752 \pm 0.116$ & $0.678 \pm 0.122$ & $0.776 \pm 0.108$ \\
\hline
\end{tabular}




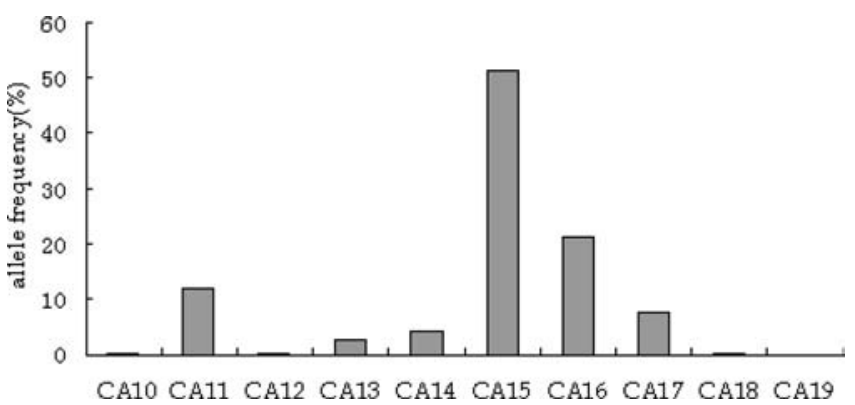

Fig. 1 Allele frequencies of the $(C A)_{n}$ polymorphism in intron 4 of the TNFR2 gene. Allele frequencies of the $(\mathrm{CA})_{\mathrm{n}}$ polymorphism, obtained from the parent group of our study subjects, are as follows: CA10, $0.28 \%$; CA11, $12.08 \%$; CA12, $0.24 \%$; CA13, $2.64 \%$; CA14, 4.32\%; CA $15,51.16 \%$; CA16, $21.32 \%$; CA 17 , $7.68 \%$; CA18, 0.24\%; CA19, 0.04\%

Table 2 Results of the QTDT analyses ( $P$-values). Femoral neck BMD values were natural logarithm transformed. BMD values were adjusted for age, height and weight. Sex was not chosen as a covariate since all the offspring were females in the nuclear families and we excluded the effects of parents' phenotypes in the QTDT. The values in parentheses are the $P$-values from the analyses of excluding 30 extremities. The value in square bracket indicates the empirical $P$-value from 1,000 permutation tests

\begin{tabular}{lll}
\hline $\begin{array}{l}\text { TNFR2 } \\
\text { alleles }\end{array}$ & $\begin{array}{l}\text { Lumbar spine } \\
\text { BMD }\end{array}$ & $\begin{array}{l}\text { Femoral neck } \\
\text { BMD }\end{array}$ \\
\hline $\begin{array}{l}\text { Population stratification } \\
\text { CA11 }\end{array}$ & $0.420(0.258)$ & \\
CA15 & $0.888(0.888)$ & $0.190(0.138)$ \\
CA16 & $0.202(0.240)$ & $0.454(0.296)$ \\
CA17 & $0.139(0.335)$ & $0.752(0.888)$ \\
Total-family association & \\
CA11 & $0.806(0.549)$ & $0.888(0.888)$ \\
CA15 & $0.017(0.010)$ & $0.807(0.590)$ \\
CA16 & $0.021(0.015)$ & $0.498(0.258)$ \\
CA17 & $1.000(1.000)$ & $0.142(0.162)$ \\
Within-family association & \\
CA11 & $0.467(0.227)$ & $0.442(0.368)$ \\
CA15 & $0.145(0.130)$ & $0.475(0.254)$ \\
CA16 & $0.009(0.004)[0.005]$ & $0.427(0.362)$ \\
CA17 & $0.297(0.285)$ & $0.120(0.102)$ \\
\hline
\end{tabular}

in our study. We selected the (CA) nolymorphism for this study based on the following reasons: (1) The TNFR2 gene extends to only about $26 \mathrm{~kb}$. The linkage disequilibrium (LD) study of this gene revealed that significant LD existed within this gene (Spotila et al. 2003). Therefore, the true functional variant (if exists) of the TNFR2 gene may be in LD with the $(\mathrm{CA})_{\mathrm{n}}$ polymorphism, and testing the $(\mathrm{CA})_{\mathrm{n}}$ polymorphism alone may be enough to test the association of the TNFR2 gene with PBD in our study. (2) A microsatellite marker contains more information when compared to a single nucleotide polymorphism (SNP) marker, which provides higher statistical power to test association with the same sample size. Among all the polymorphisms of the TNFR2 gene which have been studied (Spotila et al. 2000, 2003; Albagha et al. 2002; Tasker et al. 2004), the
$(\mathrm{CA})_{\mathrm{n}}$ polymorphism was the only microsatellite marker.

The mechanism underlying the association between the $(\mathrm{CA})_{\mathrm{n}}$ polymorphism and PBD is not well defined. TNFR2 can both exist in membrane-bound and soluble forms. It has been found that the $(\mathrm{CA})_{\mathrm{n}}$ polymorphism in intron 4 of the TNFR2 gene was correlated with the plasma soluble TNFR2 (sTNFR2) levels (Glenn et al. 2000), and sTNFR2 can modulate TNF at different levels (Aderka et al. 1992). Therefore, TNFR2 gene may mediate the response of bone cells to TNF by regulating the plasma sTNFR2 levels.

In summary, our preliminary results indicate that the (CA) $n$ polymorphism of the TNFR2 gene may be associated with LS BMD. It is the first study that suggests the TNFR2 gene may be involved in the susceptibility to osteoporosis in Chinese population. Further studies in other populations with larger sample sizes will be required to confirm the findings reported here.

Acknowledgements The study was partially supported by a key project grant (30230210), an Outstanding Young Scientist Award (30025025), a general grant (30470534) from National Science Foundation of China, three projects from Scientific Research Fund of Hunan Provincial Education Department (02A027, 03C226, 04B039) and a grant from Natural Science Foundation of Hunan Province (04JJ1004).

\section{References}

Abecasis GR, Cardon LR, Cookson WO (2000) A general test of association for quantitative traits in nuclear families. Am $\mathbf{J}$ Hum Genet 66:279-292

Abu-Amer Y, Erdmann J, Alexopoulou L, Kollias G, Ross FP, Teitelbaum SL (2000) Tumor necrosis factor receptors types 1 and 2 differentially regulate osteoclastogenesis. J Biol Chem 275:27307-27310

Aderka D, Engelmann H, Maor Y, Brakebusch C, Wallach D (1992) Stabilization of the bioactivity of tumor necrosis factor by its soluble receptors. J Exp Med 175:323-329

Albagha OM, Tasker PN, McGuigan FE, Reid DM, Ralston SH (2002) Linkage disequilibrium between polymorphisms in the human TNFRSF1B gene and their association with bone mass in perimenopausal women. Hum Mol Genet 11:2289-2295

Deng HW, Stegman MR, Davies KM, Conway T, Recker RR (1999) Genetic determination of variation and covariation of peak bone mass at the hip and spine. J Clin Densitom 2:251-263

Deng HW, Shen H, Xu FH, Deng HY, Conway T, Zhang HT, Recker RR (2002) Tests of linkage and/or association of genes for vitamin $\mathrm{D}$ receptor, osteocalcin, and parathyroid hormone with bone mineral density. J Bone Miner Res 17:678-686

Devoto M, Specchia C, Li HH, Caminis J, Tenenhouse A, Rodriguez H, Spotila LD (2001) Variance component linkage analysis indicates a QTL for femoral neck bone mineral density on chromosome 1p36. Hum Mol Genet 10:2447-2452

Glenn CL, Wang WY, Benjafield AV, Morris BJ (2000) Linkage and association of tumor necrosis factor receptor 2 locus with hypertension, hypercholesterolemia and plasma shed receptor. Hum Mol Genet 9:1943-1949

Lander ES (1996) The new genomics: global views of biology. Science 274:536-539

Li JL, Deng H, Lai DB, Xu F, Chen J, Gao G, Recker RR, Deng HW (2001) Toward high throughput genotyping: dynamic and automatic software for manipulating large-scale genotype data using fluorescently labeled dinucleotide markers. Genome Res 11:1304-1314 
McIntyre LM, Martin ER, Simonsen KL, Kaplan NL (2000) Circumventing multiple testing: a multilocus Monte Carlo approach to testing for association. Genet Epidemiol 19:18-29

O'Connell JR, Weeks DE (1998) PedCheck: a program for identification of genotype incompatibilities in linkage analysis. Am J Hum Genet 63:259-266

Spielman RS, McGinnis RE, Ewens WJ (1993) Transmission test for linkage disequilibrium: the insulin gene region and insulindependent diabetes mellitus (IDDM). Am J Hum Genet 52:506-516

Spotila LD, Rodriguez H, Koch M, Adams K, Caminis J, Tenenhouse HS, Tenenhouse A (2000) Association of a polymorphism in the TNFR2 gene with low bone mineral density. J Bone Miner Res 15:1376-1383
Spotila LD, Rodriguez H, Koch M, Tenenhouse HS, Tenenhouse A, Li H, Devoto M (2003) Association analysis of bone mineral density and single nucleotide polymorphisms in two candidate genes on chromosome 1p36. Calcif Tissue Int 73:140-146

Tasker PN, Albagha OM, Masson CB, Reid DM, Ralston SH (2004) Association between TNFRSF1B polymorphisms and bone mineral density, bone loss and fracture. Osteoporos Int 15:903-908

Yao WJ, Wu CH, Wang ST, Chang CJ, Chiu NT, Yu CY (2001) Differential changes in regional bone mineral density in healthy Chinese: age-related and sex-dependent. Calcif Tissue Int $68: 330-336$ 